\title{
Patient Education on Back Care
}

\author{
ABSTRACT: \\ Study Design: Clinical Perspective \\ Objective: To provide back care education for patients with low back pain. \\ Background: Understanding the internal and external forces the body is \\ subjected to, as well as the spine's response to these forces, can better equip \\ physiotherapists in educating patients with low back pain.
}

Methods and Measures: The focus of the clinical perspective is to provide physiotherapists with clinically sound reasoning when educating patients.

\section{Van Eck C, DPT, OCS, COMT, FAAOMPT B Physiotherapy (Cum Laude)}

1 University of Pretoria, South Africa.

Results: Providing a patient handout, educating them in how to incorporate back care knowledge into their daily activities.

Conclusion: Physiotherapists can play a significant role in empowering patients through education to take responsibility for their disability.

KEYWORDS: LOW BACK PAIN, BACK CARE, PHYSIOTHERAPY, BASE OF SUPPORT, LINE OF GRAVITY, CENTER OF GRAVITY, PATIENT EDUCATION.

\section{INTRODUCTION}

Improper load handling and body mechanics is considered risk factors for sustaining low back injury (Milanese 2004). However, it appears that any activity requiring physical exertion increases the risk for back injury (Milanese 2004). Low back pain is a reality to most people. Some people choose to function with the disability and others choose to limit their lives because of it. We as physiotherapists can empower back pain patients through education to become responsible for their disability and to live life to the fullest. The article will outline important aspects to consider when educating these patients. External forces on the body such as the center of gravity, line of gravity and base of support will influence the spine's stability and equilibrium (Neumann 2002). There are also internal forces acting on the lumbar region, including axial compression, shear and torsion (Norkin and Levangie 1992). The spine's ability to handle these forces plays a significant role in the risk of injury. Another aspect to consider is that the structure of the lumbar spine lends itself to bias flexion and extension movements (Palastanga 2006). The metabolic activity of the discs and joint capsules are very sensitive to mechanical movement (Urban and
Roberts 2004). Spinal stability is a result of the dynamic interaction between forces, the structure and the metabolic activity of tissues. Each tissue type has a specific stimulus for optimal repair (Grimsby 2004). The article will discuss the optimal stimulus for both collagen and disc repair. Finally, the author includes a suggested handout, "Back care: two basics", educating patients in how to cope with their back pain during daily activities. The handout word plays on the "back to basics" phrase. The two basic principles discussed in the handout are base of support and centre of gravity. Thus, patients can simply remember "back to basics" when lifting or moving loads during daily activities, protecting their backs from further injury by keeping the center of gravity inside the base of support.

\section{DISCUSSION}

\section{Forces acting on the human body}

External forces acting on the body plays a significant role on the equilibrium of a person. When moving, the body is subjected to complex external forces. In order to maintain a state of equilibrium against these forces, the body implements integrated neuromuscular and skeletal responses (Norkin and Levangie 1992). The body's centre of gravity (COG) is a point, anterior to $\mathrm{S} 2$, at which all mass is concentrated, and upon which gravity acts (Neumann and Nawoczenski 2002). In an asymmetrical object, the COG will be located toward the heavier end (Norkin and Levangie 1992). It can lie outside the object, but will continue to be the point on which gravity acts. The COG is dynamic and is influenced by body segment arrangement, posture and movement. The human body needs a base of support (BOS) to remain balanced (Norkin and Levangie 1992). The BOS is the contact point with the supporting surface such as a person's feet on the ground, a cane or a hand on a rail. The pull that gravity exerts on an object has force and direction (vertically downward). This line of pull is referred to as the line of gravity (LOG) (Neumann and Nawoczenski 2002). With an anterior displaced COG in a forward flexed posture, the LOG will fall outside the BOS and the person will be unstable. For stability and equilibrium, the LOG must fall within the BOS (please refer to the pictures in the patient handout). The larger the BOS and the closer

\section{Correspondence to:}

Corné van Eck

Cell: 0761370084

Email: fcvaneck@vodamail.co.za 
the COG is to that base, the greater the stability of the body (Norkin and Levangie 1992). When adding an external load to the body by either carrying or lifting it, a new COG for the combined mass exists. The new COG will shift towards the additional weight, proportional to the weight added (Norkin and Levangie 1992).

\section{Forces acting on the lumbar spine}

Forces acting on the lumbar spine include torsion, shear, viscoelastic creep (deformation) and torque (Norkin and Levangie 1992). The bony and soft tissue structures are well equipped to accommodate these forces. However, injury will occur with an overload. Axial compression is the force acting though the long axis of the spine at right angle to the discs (Norkin and Levangie 1992). During axial rotation, torsion is produced in the spinal segment (Neumann 2002). Shear forces acts on the midplane of intervertebral discs, causing adjacent vertebrae to move anterior, posterior or sideways relative to each other (Norkin and Levangie 1992). Resistance to shear, as occurs with trunk flexion, is provided by facet capsules, ligaments and discs (Milanese 2004). Forward bending into flexion displaces the COG anterior and resistance to this movement is provided by tensile strength in collagen fibres of the posterior ligaments, joint capsules, annulus fibrosus and muscular activity of core stabilizers (Grimsby 2004). Flexion also causes compression, tension and shear on discs (Norkin and Levangie 1992). The viscoelastic creep that occurs during sustained loading in forward flexion (or any other position) increases the neutral zone through elongation of the supporting structures. This reduces joint stability and places the spine at risk of injury (Milanese 2004). Therefore, the risk of injury to the intervertebral disc increases significantly with the combination of torsion, axial compression and sustained forward flexion (Norkin and Levangie 1992).

The distance of a load from the body as well as the weight of the load during lifting or load transfer has a powerful influence on the tension and compression created in the lumbar spine
(Milanese 2004). In other words, as the load moves away from the body, the lever arm of the load acting on the spine increases, significantly increasing the flexor torque at the spinal level. In order to reduce tension created by torque in the spine when a load is lifted or transferred, it has to be kept as close as possible to the body. Torque is an important consideration because of its influence on axial compression and rotation. When less torque is required from spinal musculature, compressive forces on joints are reduced and energy is conserved.

\section{The Lumbar spine}

A feature of the lumbar spine that is clinically important for the patient with low back pain is the nearly vertical orientation of the articular facets (Mercer 2004) (Neumann 2002). This orientation is in such a way that it facilitates flexion, extension and side bending movements (Mercer 2004), while limiting rotation (Singer et al 2004). Lumbar spinal movement allows for $2^{\circ}-5^{\circ}$ of rotation per level, $3^{\circ}-8^{\circ}$ of side flexion per level and $12^{\circ}-17^{\circ}$ of flexion/extension per level (Grimsby 2004). By not allowing mechanical movement patterns to occur, the metabolic integrity of the spinal segment will be affected (Urban and Roberts 2004). The metabolic and mechanical properties of discs and facet capsules depend on tension, compression, decompression and normal physiological movement patterns (Grimsby 2004). Considering the above information, patients may be ill advised, not to ever bend their backs, but rather their knees.

The nucleus pulposus (NP) and the annulus fibrosus (AF) are similar in composition, consisting of water, collagen and proteoglycans (Urban and Roberts 2004). The NP consists of collagen fibres which are thin and elastic, providing a flexible framework to resist compressive forces (Palastanga et al 2006) (Urban and Roberts 2004). The lumbar AF consists of thick and strong fibres, elongating little when stretched, providing tensile strength (Palastanga et al 2006) (Urban and Roberts 2004). The AF has lamellae orientated in different directions thus resisting diverse movement patterns (Bogduk in Mercer
2004). It is subjected to both tensile and compressive forces. During axial rotation, half of the AF resists torsion clockwise and the other half counter clockwise (Grimsby 2004). It is important to consider that the metabolic activity in the intervertebral disc is sensitive to the extra-cellular environment. For the properties of the disc to be maintained, loading and unloading is required (Urban and Roberts 2004). Without regular movement, the interior oxygen concentration will decrease and the lactic acid concentration increase, leading to disc degeneration (Urban and Roberts 2004). Not only the discs, but also cartilage and bone will be affected by reduced loading, causing rapid loss in volume, mass, and tensile as well as compressive strength (Grimsby 2004).

\section{Optimal stimulation for tissue healing}

Tissue healing is the property of several processes at work. These processes include internal factors such as the inflammatory process, metabolic activity of the specific tissue and histological influences. However, it is also influenced by external factors such as tension and movement. The optimal stimulus for the repair of collagen tissue is modified tension in the line of stress (Grimsby 2004). The tension used should not exceed the level of tension that newly formed collagen can withstand. Excessive tension will delay healing and cause micro trauma. Clinical judgment on how much tension to use is gauged by the pain response. Pain indicates that abnormal deformation of collagen is occurring and that damage is taking place (Grimsby 2004). Modified tension stimulates fibroblastic activity to produce collagen. Compared to the outcome of delayed activity, the result of modified tension is stronger collagen union, faster healing and reduced scar tissue adhesions (Grimsby 2004).

The optimal stimulus for disc repair is modified tension in the line of stress with compression and decompression (Grimsby 2004). Due to the obliquely orientated fibers of the AF, axial rotation will create the required tension, compression and decompression and thus stimulate fibroblastic 
activity to produce collagen and proteoglycans (Grimsby 2004). The disc has to be subjected to tension caused by complex diagonal movement patterns in order to heal and improve its tolerance to external tension and loads.

\section{Spinal Stability}

Stability of a spinal segment is the result of the dynamic interaction between the active (muscle), passive (structure) and neuromuscular (motor control) systems (Panjabi in Cholewicki and Silfies 2004). The active system is the dynamic component of muscle action, both concentric and eccentric. The second system includes passive spinal structures such as ligaments, facets, capsules, discs and vertebral bodies. Neuromuscular control constitutes the third system and includes the motor control that coordinates stability between the other two systems. In other words, stability will be compromised if any one of the systems does not function properly. Motor control is negatively affected after injury to either the passive or the active system. Even after complete healing to structures in the passive system, the recovery of motor control and thus stability, is not spontaneous (Singer 2004). An additional factor to consider is the complexity of neuromuscular control. When joint mechanoreceptors (type I, II and IV), located within the capsule are injured, the afferent input to muscles surrounding the joint is affected (Grimsby 2004). Delayed afferent input results in poor neuromuscular control of the segment. Neuromuscular control is essential for maintaining movement around a physiological axis. If the axis of motion for a joint is displaced, changes in the joint's kinematics will occur (Grimsby 2004). The integrity of structures in the subsystem, such as the articular cartilage, synovial capsule and intervertebral disc will be affected, leading to degenerative changes, risk of re-injury and further decrease in neuromuscular control (Grimsby 2004). Adding loads, speed, or repetitive motion will increase the demand on all three systems. Therefore, rehabilitation of motor control, both concentric and eccentric is essential for maintaining the integrity of all three systems (Grimsby 2004).

\section{CONCLUSION}

The author attempted to give the reader a clearer understanding of factors to consider in the education of low back pain patients. A patient needs to be empowered in how to take responsibility for their low back pain. It is important to consider the interaction between external and internal forces on the spine, spinal features, metabolic activity of the discs, tissue repair and spinal stability. Even though flexion and extension movements with rotation under axial load are important contributors to lumbar disc herniation, these movement patterns should be included in rehabilitation in order to repair and strengthen the injured tissue (Grimsby 2004). Rehabilitation of neuromuscular control (concentric and eccentric) is an essential component of spinal stability and as such, contributes to an optimum environment for structures in a spinal segment.

Judgment is required to balance tissue healing after injury with the negative effects that prolonged immobilization has on connective tissue quality. Physiotherapists can play a significant role in reducing the re-occurrence of low back injury by providing optimum stimuli for tissue healing, neuromuscular rehabilitation as well as patient education on back care. Patients need to understand centre of gravity, line of gravity and base of support as part of the management strategies in dealing with low back pain. Knowledge will empower a patient to balance much needed tension to structures, underlying disease processes, pain and daily activities. Following is a suggested patient handout, "Back care: two basics", educating patients in how to incorporate back care knowledge into their daily activities.

\section{PATIENT HANDOUT}

\section{Back Care: Two Basics}

"Back care: two basics", is a handout written to help you cope with your back pain during daily activities. The handout word plays on the "back to basics" phrase. The two basic principles discussed in this handout are (1) base of support and (2) centre of gravity. You can simply remember "back two basics" when lifting or transferring loads, protecting your back from further injury by keeping your center of gravity inside your base of support.

(See handout on next page)

\section{REFERENCES}

Cholewicki J, Silfies SP in Grieve's Modern Manual Therapy 2004, Grieve's Modern Manual Therapy 3rd edn. pp67-88. Elsevier Limited, London

Grimsby, O 2004 Ola Grimsby Institute Residency Course Notes. Histology chapter pp1-117. Ola Grimsby Institute, San Diego

Grimsby, O 2004 Ola Grimsby Institute Residency Course Notes. Lumber Spine chapter pp1-107. Ola Grimsby Institute, San Diego

Mercer S in Grieve's Modern Manual Therapy 2004 Grieve's Modern Manual Therapy, 3rd edn. pp9-37. Elsevier Limited, London

Milanese S in Grieve's Modern Manual Therapy 2004 Grieve's Modern Manual Therapy, 3rd edn. pp89-104. Elsevier Limited, London

Newmann DA, Nawoczenski DA 2002 Kinesiology of the Musculoskeletal System: Foundations for Physical Rehabilitation, pp56-87. Mosby Incorporated, St. Louis

Newmann DA 2002 Kinesiology of the Musculoskeletal System: Foundations for Physical Rehabilitation, pp251-310. Mosby Incorporated, St. Louis

Norkin CC, Levangie PK 1992 Joint Structure and Function: A comprehensive analysis, 2nd edn. pp8-45. F.A. Davis company, Philadelphia

Norkin CC, Levangie PK 1992 Joint Structure and Function: A comprehensive analysis, 2nd edn. pp125-169. F.A. Davis Company, Philadelphia

Palastanga N, Field D, Soames R 2006 Anatomy and Human movement: Structure and Function, 5th edn. pp471-549. Elsevier Limited, London

Urban JP, Roberts S in Grieve's Modern Manual Therapy 2004 Grieve's Modern Manual Therapy, 3rd edn. pp39-53. Elsevier Limited, London 


\section{Base of support}

The base of support is the contact points you have with a supporting surface. The contact points can be your feet, hands, knees or a cane. In the figure, the square around the feet represents the base of support.

\section{Centre of gravity *}

Your centre of gravity is a point, just below your belly button, where the body weight is concentrated. It is the point from where gravity pulls downwards. When lifting or transferring a load, its weight becomes a part of you and this will shift the center of gravity toward the load.

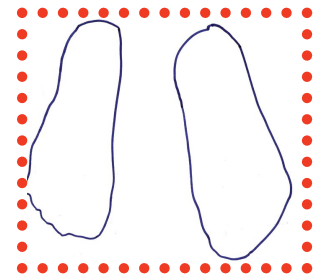

\section{Line of gravity}

It is line from your centre of gravity towards the center of the earth.

To reduce tension on the spine, discs, and muscles, it is best for this line to fall inside the base of support (See figure).
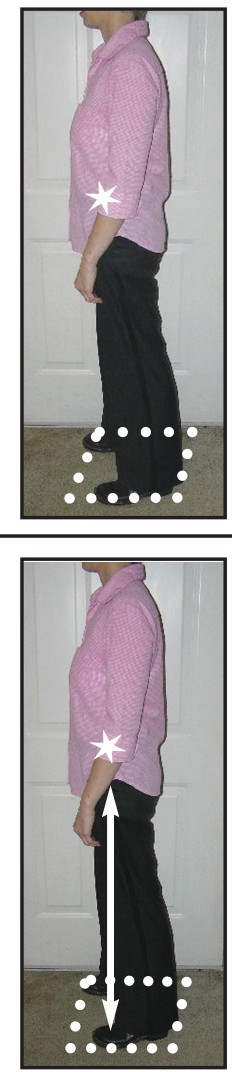

\section{Bending forward}

With bending, the centre of gravity falls outside the body and the line of gravity falls outside the base of support. This leads to higher loads and tension on the spine. Change foot position, lean on a hand, push on a knee or support your thigh against a table in order to increase the base of support allowing the centre of gravity to fall witin it.

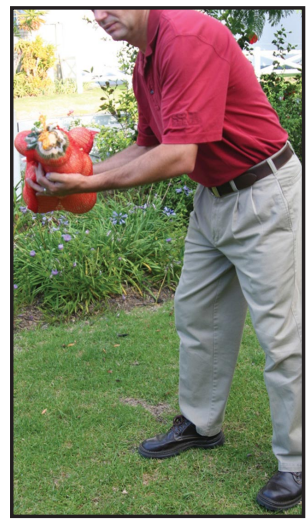

\section{Distance of the load from the body}

When lifting or shifting a load, the distance of the load from the body has a powerful influence on the stress in the spine. The further the object is away from you, the harder your back works to keep you balanced.

WRONG

Distance far from centre
CORRECT

Distance close to centre

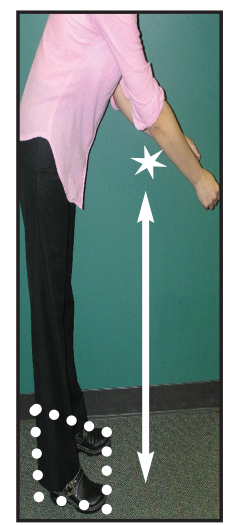




\section{SUGGESTIONS TO PERFORM ACTIVITIES OF DAILY LIVING}

Vacuuming

Keep the vacuum close to you. Step forward as you push the vacuum forward. Also applicable to lawn mowers, etc.

\section{Mopping}

Only mop the area directly around you, maintaining the contact point inside your base of support.

\section{Refrigerator}

Step "into" the refrigerator and lift the item towards you before stepping back.

\section{Making the bed}

Make the four corners individually placing a knee/hand on the bed or straighten the foot and end in the same manner.

\section{Cleaning the bath tub}

Clean the four corners individually by placing a knee/hand on the edge, or clean while you are still in the bath tub.

Washing dishes at the sink

Open the door below the sink and put your foot on a little step, brick or book inside the door.

\section{Loading and unloading}

Pull the cart close to the trunk before unloading. Place heavier items closer to you. Supporting yourself with a foot or knee on the back bumper, while loading and unloading will increase stability.

\section{Picking things up from the floor}

Position yourself to where the object you need to pick up is inside your base of support; stabilize yourself using a chair or counter if needed.

\section{Managing luggage}

Keep hand luggage as light as possible, use a suitcase on wheels versus something that needs to be carried. 


\section{Sleeping}

Sleep on a well supporting mattress, use pillows for comfort (between/underneath your knees) and alternate sides.

\section{Lifting}

When lifting an object, keep your centre of gravity inside your base of support. Position your feet in such a way as to increase the base of support.

\section{Carry a load}

Keep the load as close as possible to your body when carrying it.

\section{Pulling or pushing}

When you pull or push a heavy object, position yourself as close as possible to it. Generate power from the core muscles. Go short distances, rest and then proceed.

\section{Building}

Continue to keep objects close to your center of gravity or increase the base of support when you need to generate power in activities such as drilling, sawing, etc.

\section{PLAN ACTIVITIES AND DO NOT OVERDO THINGS WHEN FEELING BETTER}

\section{Around the House}

- Prevent repetitive motion or sustained positions exceeding 30 minutes.

- Invest in a barstool using it to occasionally sit on while preparing food or cooking.

- Conserve energy by preparing larger meals and freezing some, getting enough rest, preparing part of the meal ahead and only do a little housework each day.

- With gardening, sit on a step stool that can be moved around easily when you pull weeds or plant flowers.

POSTURE Proper posture will reduce stress on the spine.

EXERCISE Your physiotherapist will work with you to develop a progressive rehabilitation regime that will be appropriate for your level of functioning and stage of pathology. Plan the day to include exercise. When the exercises worsen your symptoms, please STOP and discuss it with your physiotherapist.

Warning signs When experiencing loss of bladder or bowel control, numbness or tingling getting worse, unexplained falling or any unexplained symptom, please do not hesitate to contact your physiotherapist or medical doctor. 